\title{
Autonomous First Response Drone-Based Smart Rescue System for Critical Situation Management in Future Wireless Networks
}

\author{
JOEL PONCHA LEMAYIAN', JEHAD M. HAMAMREH ${ }^{2}$ \\ ${ }^{1}$ Department of Electrical and Computer Engineering, Antalya Bilim University,Antalya, Turkey (e-mail: lemayian.joel@std.antalya.edu.tr) \\ ${ }^{2}$ Department of Electrical and Electronics Engineering, Antalya Bilim University,Antalya, Turkey (e-mail: jehad.hamamreh@antalya.edu.tr) \\ Corresponding author: Joel P. Lemayian (e-mail: lemayian.joel@std.antalya.edu.tr).
}

The codes used to implement the proposed system and generate the results in this article can be found at researcherstore.com

\begin{abstract}
Numerous problems have been experienced due to the current exponential rise in urban population. Such challenges include insecurity and disaster management. It is therefore critical that new and efficient ways of disaster management are realized. Drone technology has been used in many applications due to their flexibility and cost-effectiveness in executing tasks. Such applications include goods delivery, data collection, surveillance, and tracking. This paper proposes an autonomous first response drone-based (Auto-FRD) smart rescue system. Auto-FRD paradigm uses drones to provide quick response to critical situations in a smart city setting. The system comprises three main sections: sensor network, intelligent drones, and the command center. The Auto-FRD system is designed such that the drones automatically deploy to a specific location upon receiving an alert signal from the smart sensors, as opposed to people sending the signal. Moreover, the system is implemented using cheap LoRa technology. Experimental results show that the proposed system drastically reduces the response time compared to conventional critical situation response systems. Additionally, the data collected by the drones prove to be extra valuable when analyzing the critical situation.
\end{abstract}

INDEX TERMS Drones, UAV, Smart city, IoT, Wireless sensors, Emergency response, Disaster.

\section{INTRODUCTION}

C urrent technology has facilitated the integration of drones in many different application areas. [1]. Commercial companies, such as Amazon prime air, Mercedes, Dominos, and UPS have realized the benefits of drones and are utilizing them to deliver goods. Special personalized drones used for taking pictures and videos are currently available in the market. UAVs are also used by the military for surveillance and information gathering as well as executing missions over long distances. The use of drones has caught the interest of many researchers and academicians mainly because of the many advantages incurred as opposed to using conventional methods. Such advantages include low cost, safety, expandability among others. Researchers are pushing the limits of drone technology to perform new and complex functions aiming at minimizing cost and enhancing efficiency in areas such as communication and security.

As the population in cities increase, service delivery such as security becomes difficult. Currently, crisis response time is very high, and this is expected to further increase as the cities become more populated. It is therefore critical that new and effective ways are determined to mitigate this problem. Critical situations prompting new emergency response system include fire accidents, robbery, vehicle accidents, and explosions. This work explores the unique qualities of smart drones and propose a new drone system used to respond to critical situations at minimum time and provide emergency rescue services. The proposed paradigm comprises of smart drones, intelligent sensors, and drone command centers. A detailed study on drone flight planning, flight path acquisition, and permission to fly authorization will be discussed. Information management both at the 
drone and command center level will also be discussed. Additionally, a detailed study and design of the communication system comprising of camera sensors, transmitters, and receivers will also be provided.

The proposed paradigm is different compared to other related works in that, drones are automatically activated by sensors as opposed to human operators. In the event that an accident occurs, people often become very confused and are not able to observe the environment around them. This means that accurate information about the event will be very hard to obtain. Moreover, it takes a response team about 10 to 20 minutes to arrive at the accident scene. CCTV cameras have been used in many incidents to try and determine exactly what happened. Nevertheless, the cameras have many limitations such as dead zones, memory size, weather hazards, cost, and they can easily be damaged or tempered with during the accidents. The proposed system is able to quickly respond to an emergency situation, record the entire incident and even deliver required instruments such as fire extinguishers (fire extinguisher ball) or first aid items.

Some examples of real-word events where this system could have been used include, the attack that happened in Christchurch New Zealand in March 2019, which left at least 40 people dead. This was the act of a single attacker. Because the police took time to respond, he was able to do more harm. Another example is the attack that happened at Dusit hotel in Nairobi Kenya in January 2019. The police did not respond in time and even after arrival, they could not go into the building immediately to begin rescue operations. The police did not know the location of the attackers, how many they were, or what kind of weapons they had. Therefore, they spent a lot of time trying to design a rescue plan. They relied on CCTV images which took a lot of time to acquire. As a result, the attack left at least 20 people dead. Motivated by these situations and many others, the proposed system is designed and will be able to give the police quick and very crucial information that is required to save many lives. Moreover, the drones can be used to distract an attacker and give innocent people time to escape.

Some work analogous to this can be found in literature. The work proposed by [2] is closest to our proposed system. Nevertheless, the paradigm proposed in [2] faces many limitations such as unreliability and false information due to reliance on human deployment. The paradigm proposed in this work is trying to limit human influence and reduce response time. The drones are activated by distress sensor signals from smart sensors deployed in the city as opposed to people. The drones are activated by sending a global positioning system (GPS) coordinate signals. Once the drones receive this signal they immediately fly to the scene, record and send back live images. When the actual police arrive at the scene, they have access to prior information about the scene.

The information collected by the drones can be used by the police to bring the right people to justice and help prevent wrongful convictions. In Fig. 1 the use of AutoFRDs in a smart city paradigm can be observed. Drone deployment base stations are locations where drones are deployed. In the figure, it can be observed that the ideal location for a drone deployment base station is at the top of a tall building (shown by the red arrows). This is so that there will be a good line of sight (LoS), which facilitates strong communication between sensors and drones (as will be discussed in subsequent sections). Moreover, Fig. 1 shows two critical situations happening, a car accident and a building on fire. In the figure, drones hovering above the two critical situations can be observed. These drones are sending back live images to the command center and help authorities deal with the situation better.

The rest of this paper is organized as follows. Section II talks about related work. Section III presents the system model. Configurations are presented in section IV. Section $\mathrm{V}$ discusses challenges faced by the proposed system and possible solutions. Finally, the conclusion is discussed in section VI.

TABLE 1. NOMENCLATURES

\begin{tabular}{ll}
\hline \hline Abbreviations & Definitions \\
\hline BS & Base Station \\
CCTV & Closed Circuit TV \\
ESC & Electronic Speed Controller \\
FC & Flight controller \\
FC & Low-Power Wide Area Network \\
Auto-FRD & Autonomous First Response Drone \\
GA & Genetic Algorithm \\
GPS & Global Positioning System \\
HD & High Definition \\
IDE & Integrated Development Environment \\
IMM & Istanbul Metropolitan Municipality \\
IoT & Internet of Things \\
LoRa & Long-Range \\
LoRaWAN & Long-Range Wide Area Networks \\
LoS & Line of Sight \\
MIMO & Multiple Input Multiple Output \\
NLoS & No Line of Sight \\
RFM95 & Radio Frequency Modulation 95 \\
ROS & Robot Operating System \\
RSSI & Received Signal Strength Indicator \\
SA & Simulated Annealing \\
UAVs & Unmanned Aerial Vehicles \\
\hline
\end{tabular}

\section{RELATED WORKS}

The efficiency of drones in performing multiple tasks have allowed them to be used in executing many tasks with minimum cost and energy. This has attracted many researchers and engineers to employ the use of drones in many different areas. In this section, drones utilized in rescue missions, emergency cellular coverage, and accident scene mapping are highlighted.

Drones' compactness and availability have enabled their effective use in searching for survivors in collapsed buildings [3]. Authors in [3] propose a complete architecture for a rescue drone hardware. The rescue team deploy the drone at the scene and use onboard infrared 


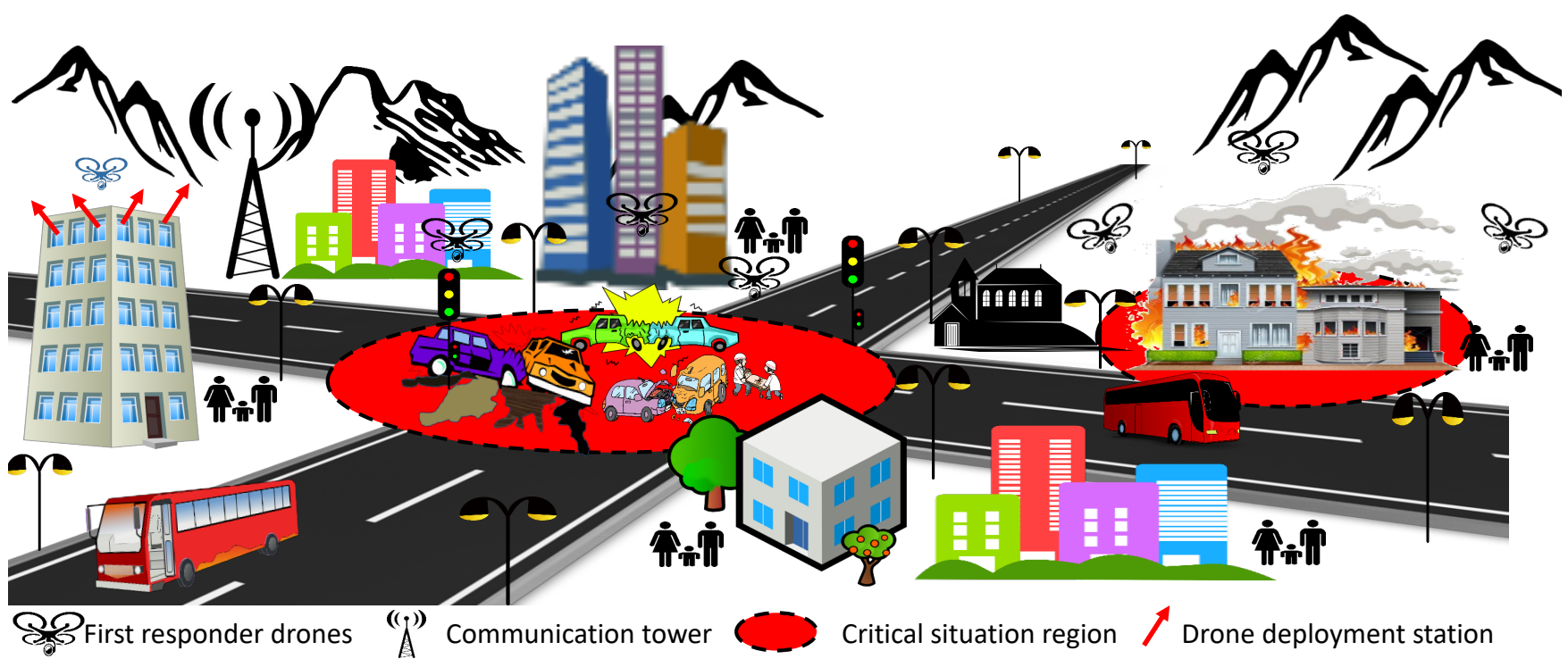

FIGURE 1. Critical situation in a smart city environment.

cameras to locate victims. This system is suitable for rescue missions in areas with no GPS accessibility. The proposed system uses, DJI Matrice 100 and hokuyo lidar, as well as Intel RealSense for global and local mapping. According to [3], results show that the proposed system performed better than conventional methods in assisting rescuers to find victims in unknown disaster affected areas.

Additionally, authors in [4] propose a paradigm where drones are deployed to provide 5G cellular coverage to a region requiring emergency cellular coverage. The region could have been affected by a disaster or too much traffic generated by users, resulting to a slow network. The goal of the system proposed in [4] is to determine the minimum number of drones required and their location for a complete coverage of the critical environment with minimum cost. While at the same time maintaining a high data rate and low latency. The authors use meta-heuristic algorithms (Simulating Annealing (SA) and Genetic Algorithm (GA)) to empirically solve the optimization problem. Moreover, authors in [5] developed a drone system to provide emergency cellular coverage in a densely populated area. Consequently, improving the performance of conventional networks.

According to the European Commission annual report in 2017, forest fires are considered among the top environmental hazards [6]. Authors in [7] propose an emergency support UAV for forest fire surveillance. The system is equipped with thermal sensors, communication modules, and thermal cameras that provide valuable realtime data about the fire to the response team. In addition, the system is implemented using multiple algorithms which automate take-off, landing, path planning, and fire monitoring. The system was simulated by performing several flight tests and the results show great performance in detecting forest fires.

Authors in [8] propose an emergency relief distribution system used after an earthquake. Arman et al. provide a detailed study of the difficulties faced by ground response after an earthquake disaster. As a result, Arman et al. propose the use of drone technology to deliver relief postearthquake to the affected people. according to [17], this paradigm is not affected by ground transportation constrains and can be used to provide services to inaccessible regions. The results show that the proposed system is very effective when providing emergency relief to affected areas.

Moreover, authors in [9] propose a top-down approach for providing post-earthquake relief using drones to residents of a large metropolitan city. The paradigm focuses on the first 48 hours after an earthquake disaster where the affected people's status and needs are analyzed and then the post-earthquake relief system is designed, and the drones deployed. The drones are deployed and controlled from a ground command center.

The related works discussed above use drones to perform different critical environment operations. The execution of the rescue plan is done when a drone is deployed by a person, or in other cases, the drone is programmed to periodically perform surveillance missions of a specific region. However, our work differs in that the drones do not require any human control to respond to a critical situation and only deploy in case an accident is detected. In many occasions, people cannot be relied to deploy the drones in a timely manner, and periodic deployment of drones is costly, wastes resources, and the drones can easily miss an accident.

For instance, the work proposed by authors in [2] is 
limited in a number of ways. First, many people may not have the drone activating-app on their phone, second, their phones may not have power. Third, if someone sends GPS signals using a phone, the done will be sent to their location which may not exactly be the place where the critical situation is happening. Fourth, people may intentionally or accidentally send a distress signal from their phones. Fifth, because of the critical situation, people may forget or may not be able to send the distress signal. The Auto-FRD system is designed in such a way that in the event of a critical situation, the drones automatically deploy at minimum time possible to the exact location of the accident.

\section{SYSTEM MODEL}

Fig. 2 describes the system layout of this project. This paradigm is composed of three main parts: the command center, intelligent drones, and smart sensors. The proposed system functions as follows, When the smart sensors located in the streets sense a critical situation such as an explosion or fire, they send GPS signals of the location to the drones. The drones which are constantly on standby automatically fly to this location and send live data to the command center or deliver services. The command center work with the police and can also take control of the drones after arriving at the scene. Each of these sections shall be briefly discussed to provide readers with a clear understanding of this model.

\section{A. ALERT SIGNAL SYSTEM}

The Alert signal system comprises of the camera system with the ability to detect a range of critical situations and a signal transmission system used to send an alert message to the drone. In this section, configuration of both parts used in this system shall be discussed.

\section{1) Sensors and Intelligent camera setup}

The intelligent cameras are mounted on locations such streets lights, buildings, and towers as shown in Fig. 3 (Currently, there are thousands of cameras around cities, which can easily be integrated with this system).The cameras have a 360 degree vision of the surrounding environment and can accurately detect an accident $3.5 \mathrm{~km}$ away. The cameras detect the accident and determine the GPS coordinates at the center of the accident. Unusual activity that can be detected by the cameras includes explosions, commotions, fires, and other forms of accidents. The authors in [10] propose an intelligent videosurveillance system with the ability to observe abnormal behavior in crowed areas and set off an alarm system. Additionally, other distress signals can be manually triggered by employees in places such as banks or other business areas in case of an emergency. Fire detectors can also be utilized to deploy drones in case of a fire accident. For example, bush fires can be hard to control. Therefore, Auto-FRDs can be used to get a clear picture of the situation and gather information such as people who need immediate evacuation.

A reliable and inexpensive communication paradigm is critical to the efficient operation of the Auto-FRD system. Therefore, the system will be implemented using Long Range (LoRa) communication system, which is enabled by Low Power-Wide Area Network (LP-WAN). Moreover, an ESP32 board shall be used to interface the GPS module with LoRa and other sensors. The details of these hardware devices used are given below.

\section{2) Alert signal transmission using ESP32-LORA system}

LoRa is a long-range wireless communication technology promoted by the LoRa Alliance. It uses radio modulation systems to transmit data. The modulation technique used for communication in LoRa allows for long-range transmission of small data packets. This means low bandwidth consumption. The complexity of a LoRa receiver is also reduced considerably due to the similarity of the offset in time and frequency between the sender and receiver [22] Moreover, there is minimum interference and low power consumption. These qualities equip LoRa for a plethora of applications in a smart environment setting [11]. In addition, LoRa uses unlicensed frequencies that are freely accessible and can be used by anyone.

LoRa's long-range and low power features make it perfect for battery-operated sensors and low-power applications in the Internet of Things (IoT), Smart home and Machine-to-machine communication. There are two different topologies for LoRa communication paradigm. Point to point communication and LoRa network (using LoRaWAN for example) as seen in Fig. 4 and 5 respectively.

Unlike WiFi or Bluetooth that only support shortdistance communication, two LoRa devices with a proper antenna can exchange data over a long distance. You can easily configure your ESP32 with a LoRa chip to transmit and receive data reliably at $2-5 \mathrm{~km}$ in an urban environment and up to $45 \mathrm{~km}$ diameter in rural areas [11].

This system supports key Internet of Things (IoT) requirements such as bi-directional communication, endto-end security, mobility and localization of services. LoRaWAN has gained a lot of popularity among researchers and academicians. Especially in this age of IoT where billions of things are connected to everything and power consumption becomes a major concern [12]. Table II highlights comparisons between LoRaWAN and other LPWA communication technologies. As can be observed from the table, LoRaWAN is fairly superior in terms of data rate, battery life, interference immunity, and mobility.

Because of the above mentioned reasons, LoRa technology with ES32-RMF95 modules is used to build the communication system between the intelligent sensors deployed in the streets and the standby drones. This link is used to send alert signals. The algorithm used in 


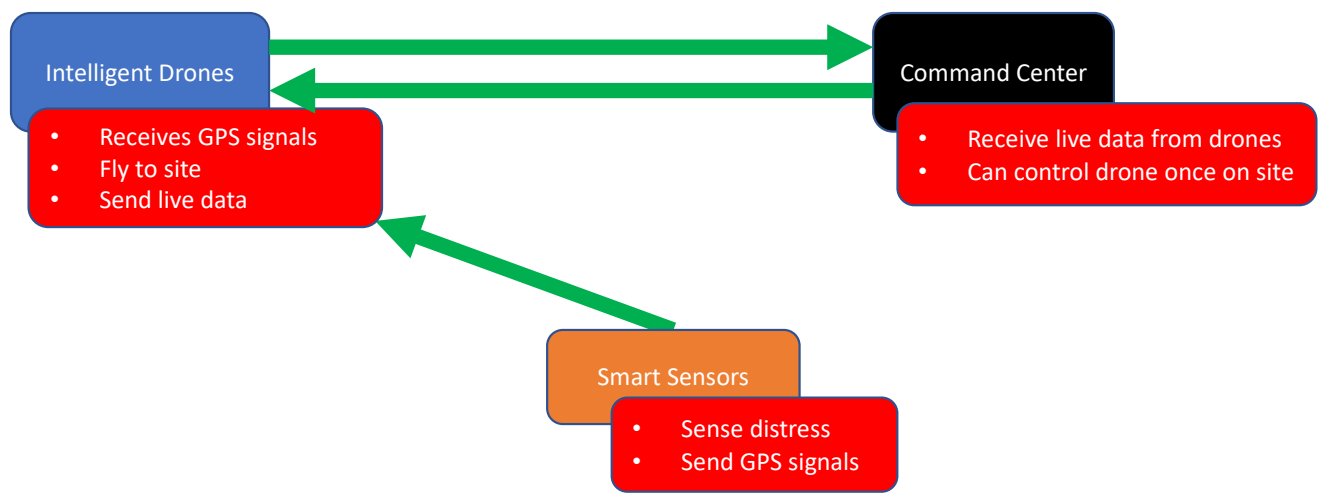

FIGURE 2. System layout of the proposed first response drone-based emergency system.

TABLE 2. LoRaWAN and others LPWAN communication technologies

\begin{tabular}{|c|c|c|c|c|}
\hline Feature & LoRaWAN & Sigfox & NB-IoT & LTE-M \\
\hline Modulation & SS Chirp & GFSK/DBPSK & UNB/GFSK/BPSK & OFDMA \\
\hline Data Rate & 290bps -5ookbps & 100bps $12 / 8$ bytes Max & 100bps $12 / 8$ bytes Max & 200kbps-1Mbps \\
\hline Link Budget & $154 \mathrm{~dB}$ & $146 \mathrm{~dB}$ & $151 \mathrm{~dB}$ & $146 \mathrm{~dB}$ \\
\hline Battery Lifetime & $8-10$ years & 7 - 8 years & $7-8$ years & $1-12$ years \\
\hline Power Efficiency & Very High & Very High & Very High & Medium \\
\hline Security/Authentication & $\begin{array}{l}\text { Yes (32 bits) } \\
2-5 \mathrm{~km} \text { urban }\end{array}$ & $\begin{array}{l}\text { Yes (16 bits) } \\
23-10 \mathrm{~km} \text { urban }\end{array}$ & No $1.5 \mathrm{~km}$ urban & $\begin{array}{l}\text { Yes (32 bits) } \\
35 \mathrm{~km}-2 \mathrm{G}\end{array}$ \\
\hline Range & $\begin{array}{l}15 \mathrm{~km} \text { suburban } \\
45 \mathrm{~km} \text { rural }\end{array}$ & 30-50 km rural & 20-40 km rural & $\begin{array}{l}200 \mathrm{~km}-3 G \\
200 \mathrm{~km}-4 G\end{array}$ \\
\hline Interference Immunity & Very High & Low & Low & Medium \\
\hline Scalability & Yes & Yes & Yes & Yes \\
\hline Mobility/Localization & Yes & No & Limited.No Loc & Only Mobility \\
\hline
\end{tabular}

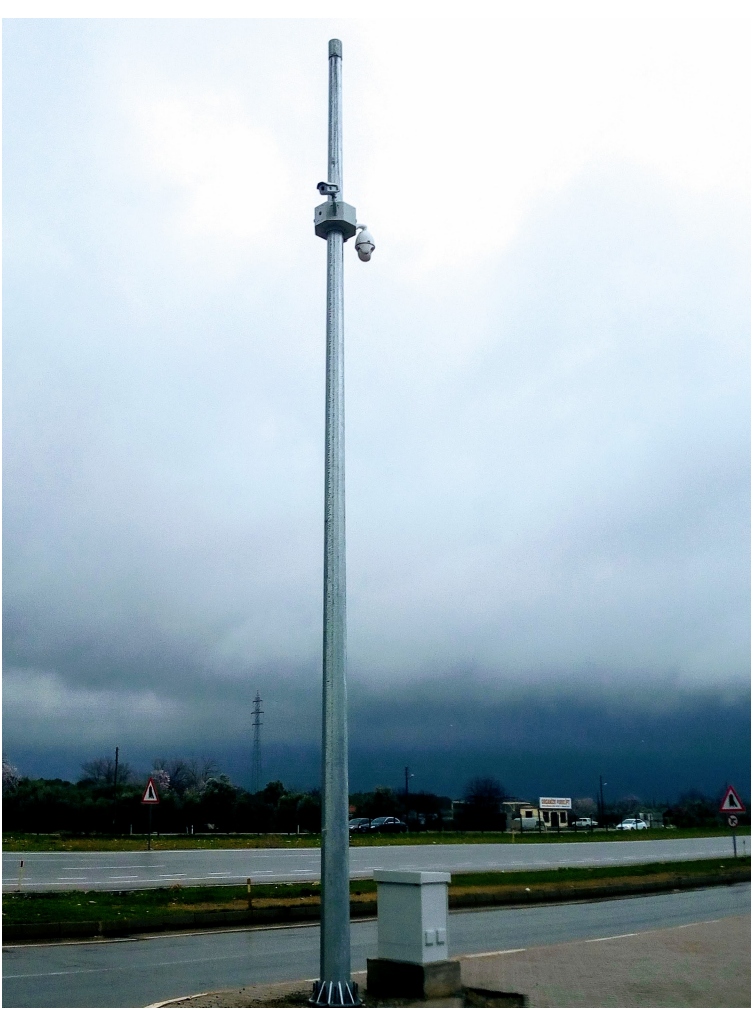

FIGURE 3. Surveillance intelligent camera system mounted on the streets.
D1

FIGURE 4. Long range device to device communication.

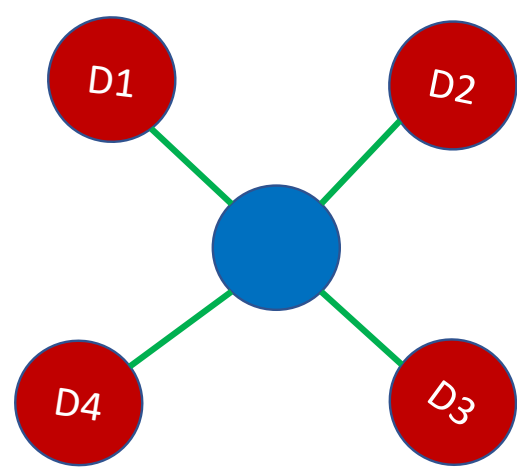

FIGURE 5. Long range wide area network (LoRaWAN) guarantees perfect operation between loT devices without complex local implementation

the transmitter is depicted in algorithm 1. The algorithm shows that the transmitter sends multiple alert GPS coordinate signals when activated. This is to ensure that the packets have a high delivery rate. The communication system was tested by mounting the receiver on high ground and moving the transmitter to different locations 
as seen in Fig. 6.

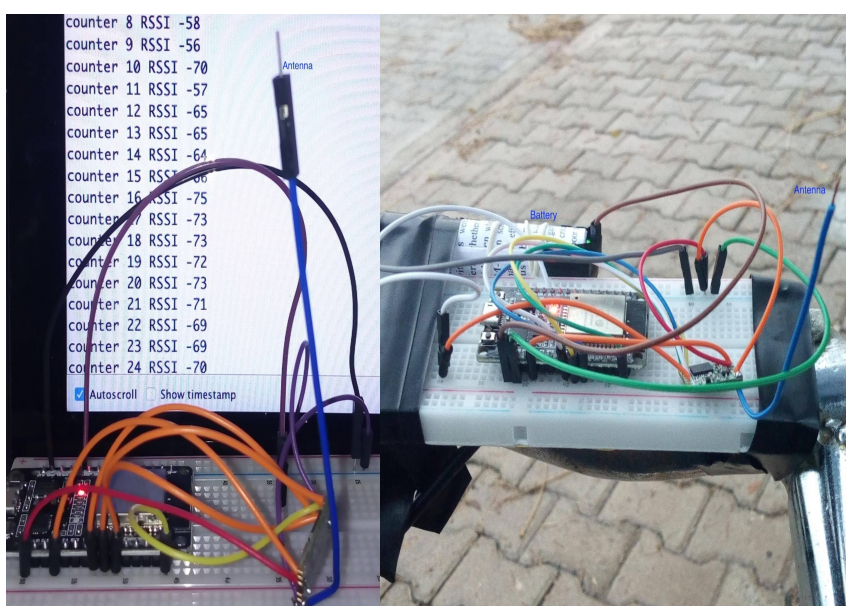

FIGURE 6. Signal receiver (left) depicting the RSSI of the packet and mobile signal transmitter (right) mounted on a bicycle.

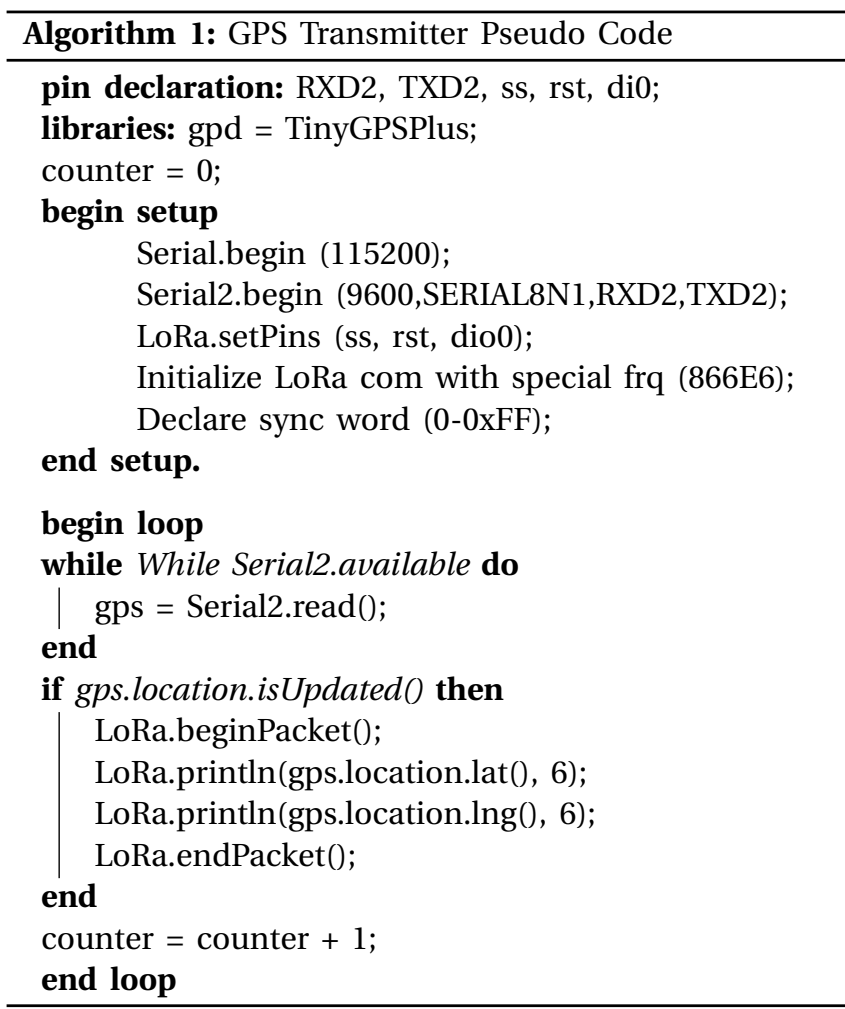

Fig. 7 shows the different locations where the transmitter was placed. These places, A through to E, were strategically chosen to measure coverage, as well as RSSI of LoRa on this system under different conditions. Location A has direct LoS, location B has the most dynamic environment due to moving vehicles and location $\mathrm{D}$ has the highest interference and distance from the transmitter. RSSI was measured and compared for LoS and NLoS from positions A, C, and E and findings depicted in Fig. 8. From the figure, it can be observed that the RSSI for NLoS is weaker than that of LoS. Nevertheless, as the distance increases, the RSSI for both

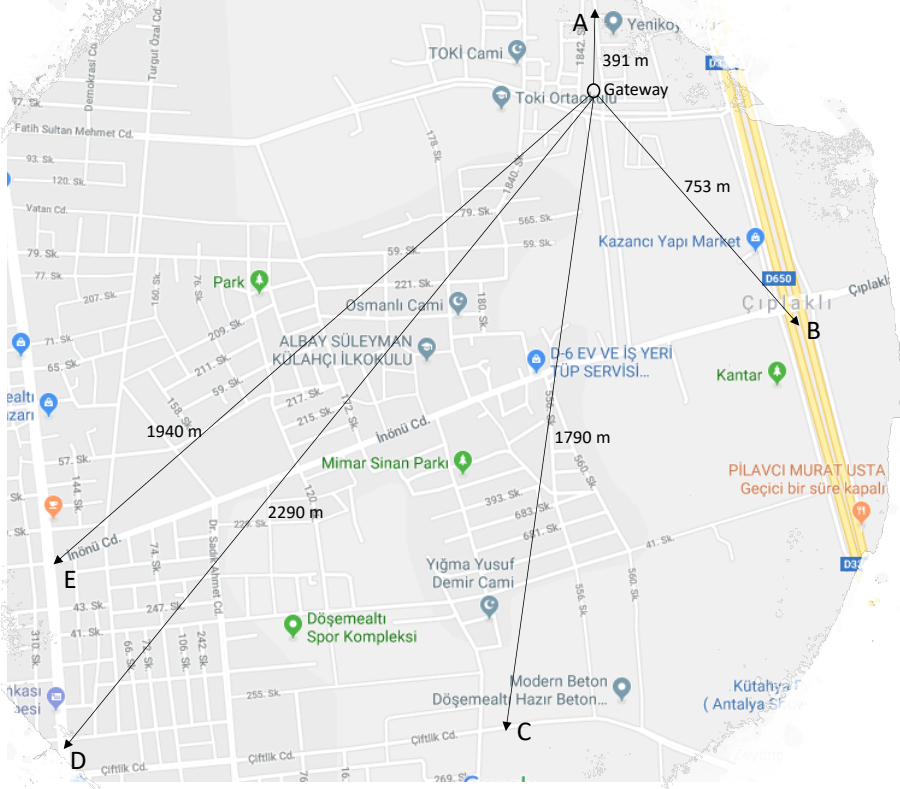

FIGURE 7. Map containing locations used to obtain LoRa RSSI field test data

LoS and NLoS converge. Moreover, the rate of received packets was measured from all locations and represented in Fig. 9. The figure shows a continued decent in the rate of received packages, with the highest being almost $100 \%$ and the lowest $55 \%$ at positions A and D respectively. It is determined that communication between the transmitter and receiver in a LoRa system is dependent on the distance and interference encountered by the system. The higher the interference the lower the RSSI. Moreover, the longer the distance, the lower the packet delivery rate.

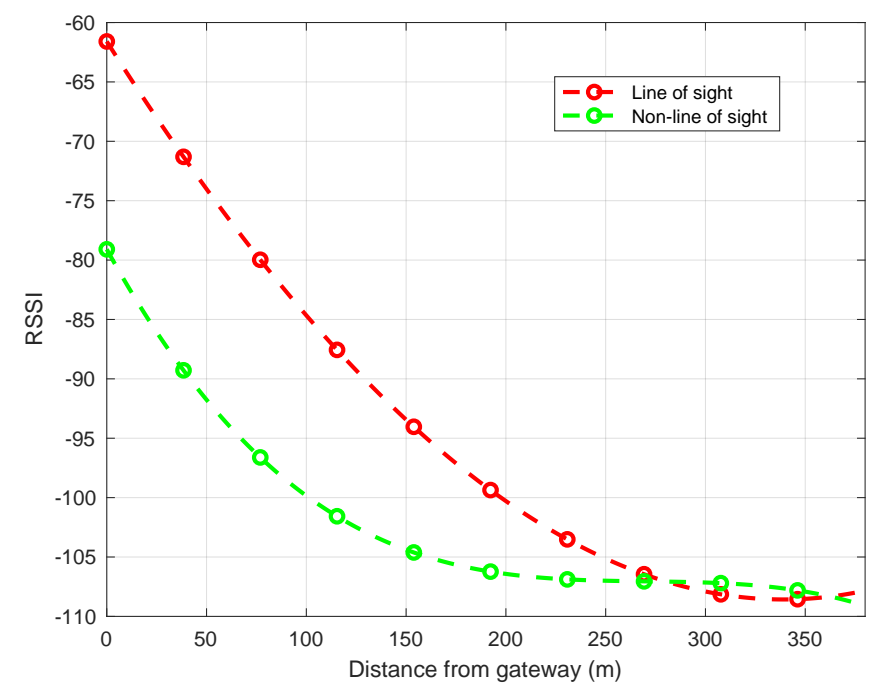

FIGURE 8. RSSI for LoS and NLos as the distance from gateway increases. 


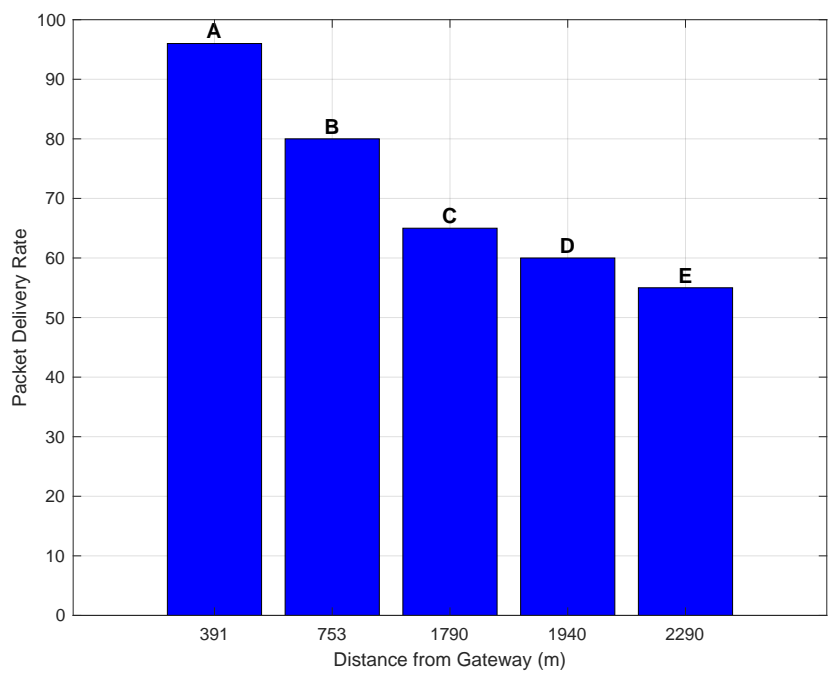

FIGURE 9. The rate of packet delivery as distance between gateway and transmitter increases as well as obstacles.

\section{B. DRONES}

Modern-day technology has allowed for the development of intelligent high functioning drones. Autonomous drones with the ability to function with minimum human control exist in the market today. For instance, Amazon drones are pre-programmed to fly to the customer and back without human intervention. They are also equipped with an anti-collision algorithm. This means that they can avoid colliding with each other or with other objects. Moreover, when the drone loose connection with the control station, they automatically return to a secure location. In this work, the abilities of these intelligent drones are exploited to develop an Auto-FRD system.

Different factors were considered in determining the type of drone to be used in this work. Such aspects include payload, cost, weather, maneuverability, and power consumption. A hexacopter shown in Fig. 11 was therefore chosen as a better candidate as it is more stable and can withstand different weather elements compared to other models. Table III summarises the properties and components of the hexacopter. Other components used include optical cameras, high precision sensors (RTK GNSS, accelerator, and gyroscope) for precise data collection (speed, orientation, gravity) as shown in Fig. 10. Take-off, path planning and permission to fly procedures will be discussed in this section.

TABLE 3. Drone properties

\begin{tabular}{cc}
\hline \hline Properties and components & Quantity \\
\hline Take-off mass & $8.2 \mathrm{~kg}$ \\
Flying top speed & $32 \mathrm{~m} / \mathrm{s}$ \\
Endurance & $18 \mathrm{mins}$ \\
flight controller & PixHawk $2 \mathrm{Cube}$ \\
Dimensions & $1250 \mathrm{~mm} \times 1250 \mathrm{~mm} \times 730 \mathrm{~mm}$ \\
\hline
\end{tabular}

When the drone located at base station $\left(x_{0}, y_{0}\right)$ receives an alert signal at $\left(x_{e}, y_{e}\right)$ which is the location of an emergency, the first thing the drone does is to request permission to fly by sending locations $\left(x_{0}, y_{0}\right)$ and $\left(x_{e}, y_{e}\right)$ to the traffic control center. Ones the permission to fly is granted, the drone then takes off to a safe altitude $h$ allowed by the city's drone flying regulations.

From point $\mathrm{o}=\left(x_{0}, y_{0}, h_{o}\right)$ the path finding algorithm finds the path with the lowest probability of collision to $p_{e}=\left(x_{e}, y_{e}, h_{e}\right)$. The algorithm considers three potential paths used by the drone to arrive at the final destination. From Fig. 12, the drone can use path $o-p_{e}, o-o_{l}-p_{e}$, or $o-o_{r}-p_{e}$.

First, the path finding algorithm calculates a safety coefficient $k_{o-p e}$ of path $o-p_{e}$. Then, locations $o_{l}$ and $o_{r}$ are calculated depending on the location of the accident, by considering the furthest points with no obstacles from $\left(x_{0}, y_{0}, h_{o}\right)$, and the algorithm also allocates a safety coefficient $k_{o-o l}$ and $k_{o-o r}$ which determines if the drone will choose point $o_{l}$ or $o_{r}$. The algorithm then calculates the safety coefficient from the chosen point to $\left(x_{e}, y_{e}, h_{e}\right)$.

Finally, the algorithm determines the path to be used by the drone by choosing the path with the highest safety coefficient.

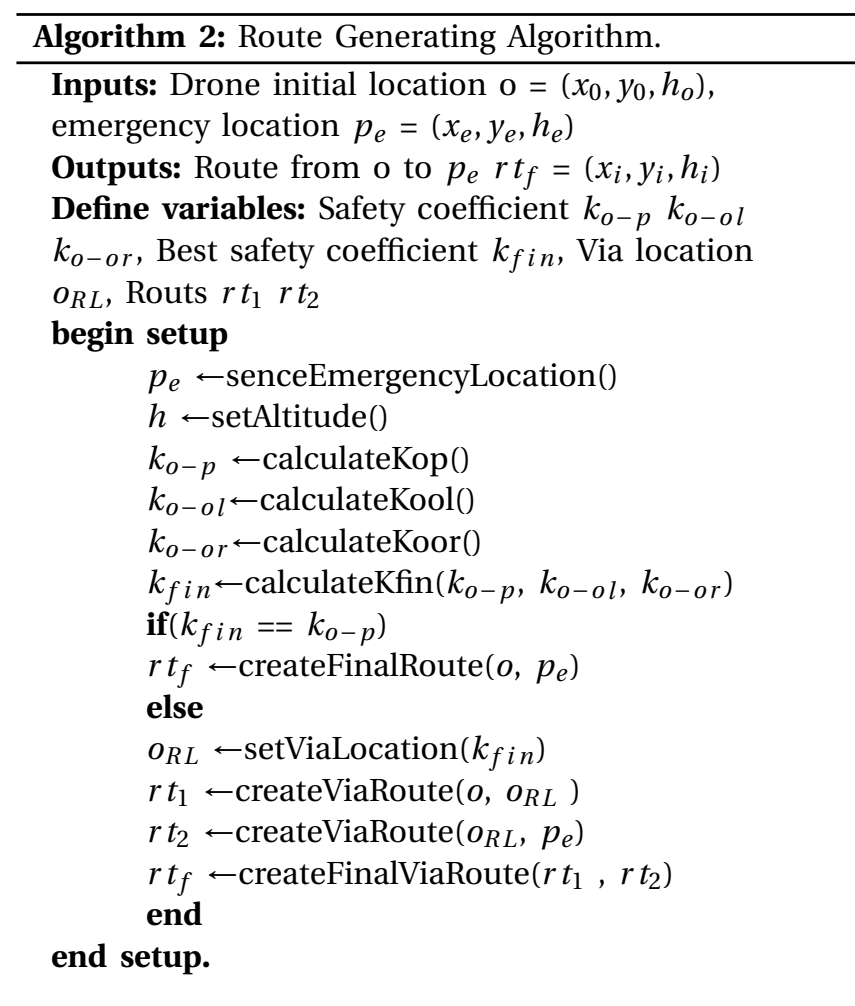

\section{COMMAND CENTER}

A graphical user interface (GUI) is developed at the command station to allow personnel to control and monitor drone activity while at the scene. The GUI is basically divided into two main sections. The first section is used to establish a connection between the on-board computer and the base while the second is used to visualize the on- 


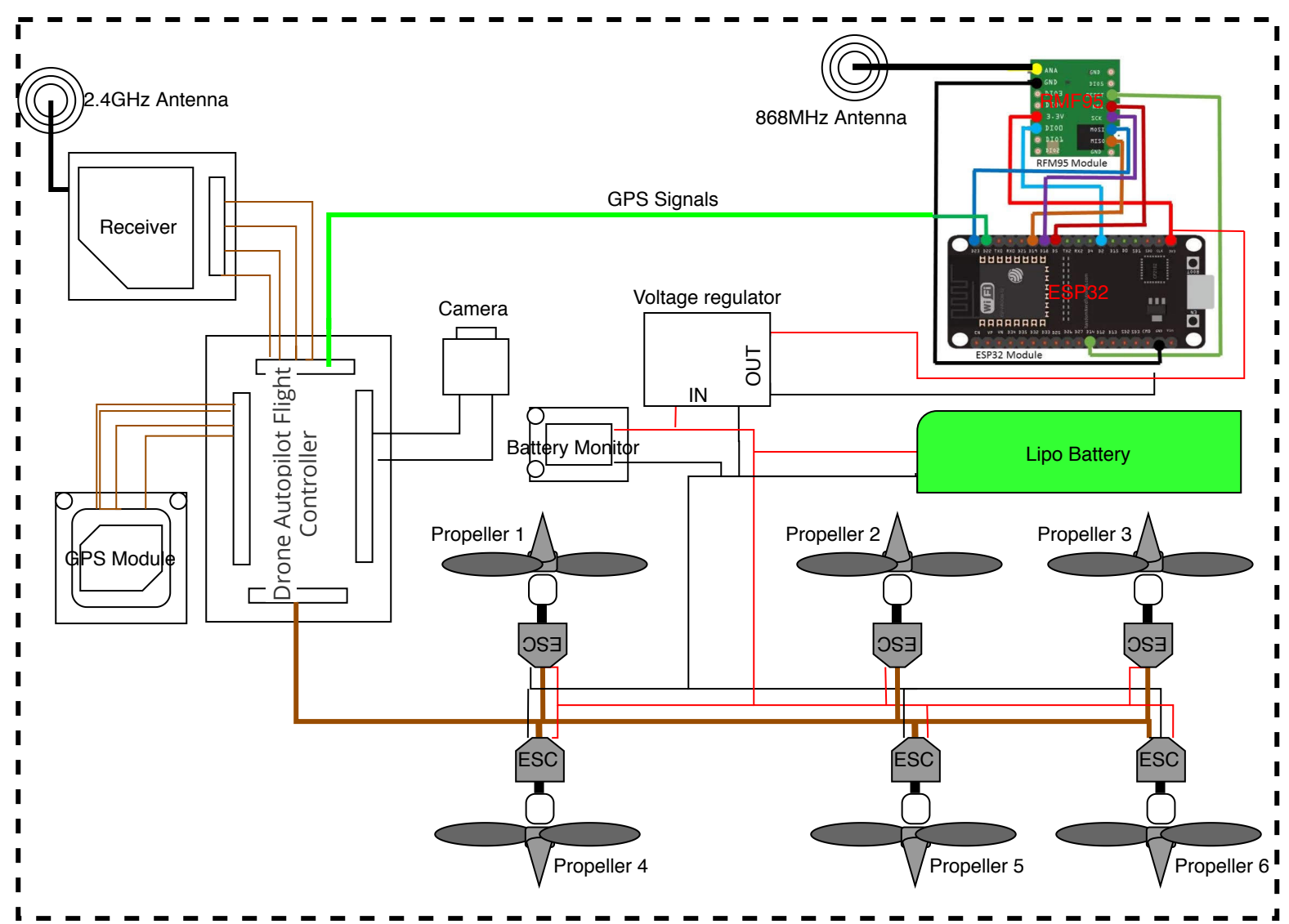

FIGURE 10. Proposed drone paradigm incorporating the GPS receiver for Auto-FRDs.

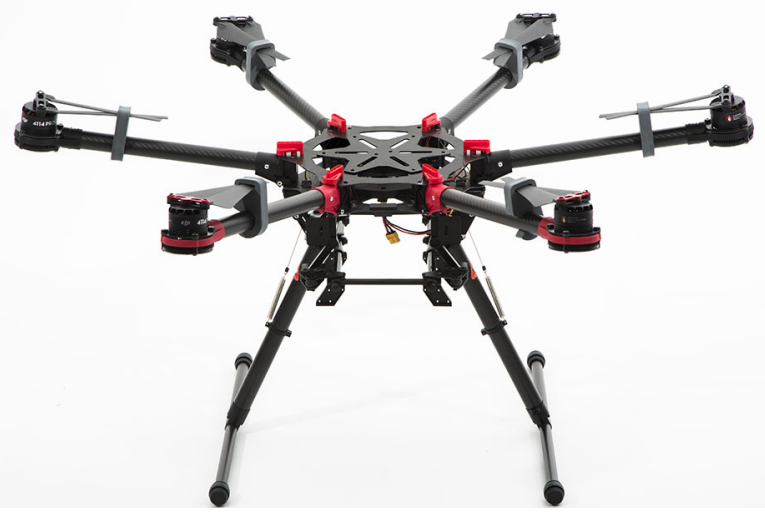

FIGURE 11. Auto-FRD model.

board sensor readings. The second part is further divided into the following sections:

- Optical sensors: This section displays the live video signal from the HD camera on the drone.

- Autopilot information: This section displays all the information about the drone from the autopilot. Such information include drone speed, altitude, battery life, and other information about the status of the flight.

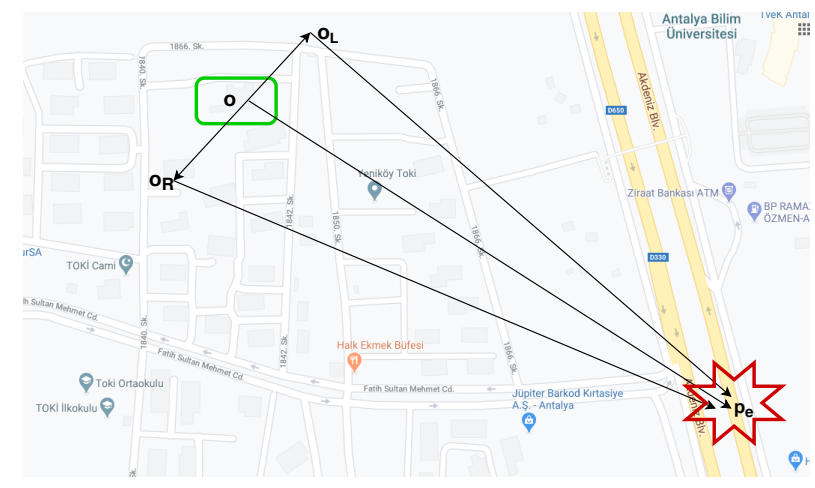

FIGURE 12. Map containing locations used to obtain LoRa RSSI field test data.

- Drone position on map: This section shows the location of the drone as well as the path of the drone on a satellite mode map.

As noted by the authors in [13], human labor is still required despite the high level of programming in autonomous smart drones. People are required to monitor the flight path of the drones as well as maintain the drones. In this system, the command centers are used for this purpose. Moreover, when the drones arrive at the critical environment scene, they send live signals to the 
station. The command centers can therefore, collaborate with the police to provide urgent and valuable information about the scene.

\section{CONFIGURATIONS}

The Auto-FRD system is designed to automatically provide fast services to people in need. Therefore, the time used by the proposed system to provide the emergency service must be compared against conventional methods. The speed of a smart drone is mostly dependent on variables such as wind speed and direction, and payload of the drone. In this work, the top speed archived by the designed drone is approximately $32 \mathrm{~m} / \mathrm{s}$

Therefore, for a distress signal $5 \mathrm{~km}$ away from the drone deployment base station, the Auto-FRD would take less than 3 minutes to get to the scene. On the other hand, the time used by the police to respond to a distress call is affected by many variables. The day of the week is one variable that affects police response time. The authors in [14] claim that the police workload is higher on Friday and Saturday than any other day of the week. Therefore, they take a longer time to respond to distress calls. Moreover, the time of the day (night-time or day-time), location of the incident (characteristics of the neighborhood) and the type of the incident are the other factors affecting police response time. According to a study done by [14] on the response time by police to a burglary in progress in Texas USA, about $75 \%$ of the calls were responded to in less than 5 minutes. Wheres, almost $5 \%$ took more than 10 minutes. In this section, a case study of implementing the proposed model in one of the most crowded city in the world, Istanbul in Turkey was done.

Istanbul is the fifth largest Megacity in the world [15]. Spanning over two continents (Europe and Asia), the city holds a population of over 15 million people [15]. As a result, the city is facing problems such as traffic congestion, insecurity, and insufficient fire station to meet the needs of its residents. According to the Istanbul fire department, there are about 70 fire stations currently operating in the city. The stations range from small outposts known as squads, to big main fire centers [16]. Each station covers an area of about $80 \mathrm{~km}^{2}$. Many fire stations have been developed by the Istanbul metropolitan municipality (IMM) in an effort to provide emergency service to the large population of the city [16]. The average response time is calculated using the equation below.

$$
\tau=d / u+x+\delta_{t}\left(\mu_{t}, \alpha_{t}\right)+y+\delta_{r}\left(\mu_{r}, \alpha_{r}\right)
$$

Where, $d$ is the distance between the fire station and the emergency location, $u$ is the speed of the vehicle. $x$ and $y$ are the average delay caused by the traffic and fire fighters respectively. $\delta_{t}$ is the variation delay caused by road traffic as well as the delay due to the location of the fire accident (Such as narrow pathways). $\delta_{r}$ is the variation delay caused by the personnel when getting ready. $\delta_{t}$ and $\delta_{r}$ are uniform distribution with mean $\mu$ and variance $\alpha$, this is because is because the exact delay caused by the two events is not constant but varies with $\pm \alpha . \mu_{t}$ and $\alpha_{t}$ are determined by the traffic delay during peak and off-peak hours in the city. While $\mu_{r}$ and $\alpha_{r}$ are determined by how ready the firefighter personnel are during the emergency call. Using the proposed drone system, $\delta_{t}$ is the effect of air resistance and wind on the drone and $\delta_{r}$ is the time taken to send and receive permission to fly.

The average speed of an emergency vehicle is dependent on many factors such as the condition of the road and the driver. In this study, it is assumed that these factors cause negligible delay and that the fire truck is able to maintain an average speed of $17 \mathrm{~m} / \mathrm{s}$ which is the common speed maintained by firetrucks during an emergency [17]. Equations 1 is used to compute and compare the emergency response time of both systems. Fig. 13 depicts the performance of the fire department in Istanbul compared to the proposed Auto-FRD system. As it can be observed, the proposed system performs better by having a lower response time. In addition, since drones cover a large area in a very short time, only a few base stations are required to cover the city as opposed to having many fire stations and personnel, and hence save money.

Table IV compares the performance metrics of the proposed system with conventional police response system and related drone paradigms like these covered in the related work section (referred here as other drone paradigm). The metrics considered included the monthly cost of implementing and maintaining the system. There are two methods used by the police to reduce response time to a critical situation [18]. The first method is hiring one additional response officer (M1) and the second is the relocation of response stations (M2). From the table, it can be observed that the Auto-FRD system is cheaper compared to other drone response paradigms such as those proposed in [2], [19], and [20], as well as the police emergency response system. The response time is the average time taken for a response service to arrive at the emergency scene.

On average, the police take 4 minutes to respond to an emergency with a 2.66-minute standard deviation [14]. Most other emergency response drones paradigms require people to deploy them. Therefore, their response time is greater than the proposed Auto-FRD system but less than the conventional police methods. Reliability metric measures if the system delivers when called upon. Police response systems are limited by many factors such as traffic jam, police ethics (ie. some police may choose to ignore an emergency call), and availability of personnel, hence they may not always deliver. On the other hand, systems that use drones are more reliable as they have fewer limitations and are more likely to 


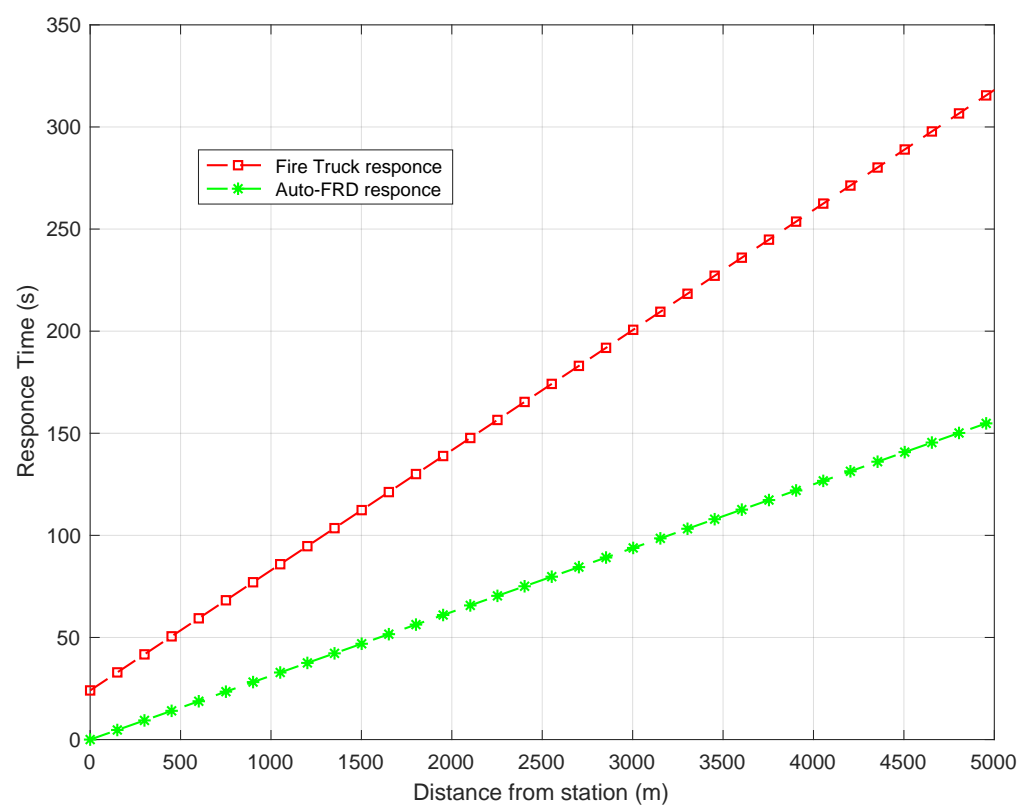

FIGURE 13. Performance of the proposed Auto-FRD system Vs Istanbul Fire Department

respond. Complexity is the amount of effort needed to request an emergency service. For the police system, a person is required to make a phone call and request a service. For other drone systems, a person is required to press an alarm signal [2]. However, in the proposed AutoFRD system, people are not required to do anything, and hence it has the lowest complexity compared to the other systems.

False alarm rate is the measure of false requests for an emergency. As noted by [21], the emergency police response unit receives a high number of false calls which cost time, money and put lives in danger. These calls are deliberately made with criminal motives such as crime concealment, revenge, or terror. Other drone systems where people press emergency signals can be used in the same way as making a false emergency call to the police, moreover, one must acquire the application to send the signal in addition to having a phone. On the other hand, Since the proposed system uses smart sensors to send a distress signal, the rate of error observation is low compared to the other methods. Nevertheless, If the sensors are not trained well to identify emergency situations, a false signal may be sent. However, this mistake can easily be rectified by using well-trained sensors.

The accuracy of information obtained measures how well the data obtained can reflect the exact emergency situation. Using drones provide video and picture evidence, as opposed to obtaining a descriptive account of the situation by the witnesses which is what happens in many police response system. Volume of information obtained measures the amount of information collected about the critical event. This is greatly affected by the response time because the lower the response time, the more an emergency can be observed and obtain more data. The automation metric shows the response system's human dependence. Most police response systems have limited or no automation. Also, many emergency drone systems require some form of human interaction before they are deployed. However, the proposed AutoFRD system is fully automated during an emergency response situation. According to the performance metrics considered in Table IV, the proposed paradigm is better than the other emergency response systems.

TABLE 4. Performance metrics of the proposed system compared to other emergency situation response systems.

\begin{tabular}{|c|c|c|c|}
\hline $\begin{array}{l}\text { Measurement } \\
\text { Metric }\end{array}$ & Auto-FRD System & Police System & $\begin{array}{l}\text { Other drone } \\
\text { systems (eg. [22]) }\end{array}$ \\
\hline Cost & $\approx 300.00 \$$ & $\begin{array}{l}\mathrm{M} 1 \approx 5082 \$ \\
\mathrm{M} 2 \approx 41,078.44 \$\end{array}$ & $\approx 8,870.00 \$$ \\
\hline Response Time (s) & $\approx 180$ & $\approx 525$ & $180<$ time $<525$ \\
\hline Reliability & High & Medium & High \\
\hline Complexity & Low & Medium & High \\
\hline False alarm Rate & Low & High & Medium \\
\hline $\begin{array}{l}\text { Accuracy of } \\
\text { information } \\
\text { obtained } \\
\text { Volume of }\end{array}$ & High & Medium & High \\
\hline $\begin{array}{l}\text { Information } \\
\text { obtained }\end{array}$ & High & Low & Medium \\
\hline Automation & Fully & None & Semi \\
\hline
\end{tabular}

\section{CHALLENGES}

Even though the autonomous first response drone-based paradigm proves to be effective in handling critical situations, the system still faces some challenges. One limitation is the unreliability nature of the GPS module to provide accurate location coordinates. GPS signals can be critically weakened by the surrounding environ- 
ment which blocks the satellite link. Such environmental obstacles include tall buildings, tunnels, tree cover, and weather elements such as clouds and humidity. However, proposed solutions to overcome this challenge include the use of satellite networks such as the Russian GLONASS network. There are GPS modules that can communicate with more than one GPS network. This means that the GPS receiver can pick up multiple satellite signals and improve its reliability and performance. Modules such as the Ublox Neo 7N and Ublox Neo M8N 436 modules support both GPS and GLONASS networks. Moreover, optic flow which is a vision-based motion estimation system can be used to decrease the GPS location errors [22]. Drones like DJI Phantom 3, and Inspire one 155 have inbuilt Optic flow features, that allows them to fly indoors.

Another challenge faced by the Auto-FRD paradigm is the loss of transmitted packets. As shown by Fig.s 8 and 9, location coordinates send from the transmitter can fail to reach the receiver due to NLoS and distance. Proposed solutions to combat this challenge include the use of MIMO (Multiple transmitter and receiver antennas) to increase the path of the signals, consequently increasing the rate of packets received at the receiver.

\section{CONCLUSION.}

This study shows that using Autonomous First Response Drones-based smart rescue system for critical situation management is a novel idea that can be used to save many lives by significantly reducing emergency response time. In addition, the paradigm is inexpensive, secure and reliable compared to conventional response-timereduction methods. Future works of this project include implementing a network of drones and sensors using LoRaWAN by further utilizing IoT systems and smarter city paradigms. Moreover, we intend to modify the drones to autonomously deliver more services to a critical situation such as fire extinguishing balls.

\section{The authors declare no potential conflict of interest}

\section{REFERENCES}

[1] A. Fadi, J. Lemayian, S. Alturjman, and L. Mostarda. "Enhanced Deployment Strategy for the 5G Drone-BS Using Artificial Intelligence." IEEE Access 7 (2019): 75999-76008.

[2] Ermacora, Gabriele, Antonio Toma, Stefano Rosa, Basilio Bona, Marcello Chiaberge, Mario Silvagni, Marco Gaspardone, and Roberto Antonini. "A cloud based service for management and planning of autonomous UAV missions in smart city scenarios." In International Workshop on Modelling and Simulation for Autonomous Systems, pp. 20-26. Springer, Cham, 2014

[3] L. Seoungjun, D. Har, and D. Kum. "Drone-Assisted Disaster Management: Finding Victims via Infrared Camera and Lidar Sensor Fusion." In 2016 3rd Asia-Pacific World Congress on Computer Science and Engineering (APWC on CSE), pp. 84-89. IEEE, 2016.

[4] Al-Turjman, Fadi, Joel Poncha Lemayian, Sinem Alturjman, and Leonardo Mostarda. "Optimal Placement for 5G Drone-BS Using SA and GA." In Drones in IoT-enabled Spaces, pp. 43-58. CRC Press, 2019.

[5] Li, Xiaowei, Haipeng Yao, Jingjing Wang, Xiaobin Xu, Chunxiao Jiang, and Lajos Hanzo. "A Near-Optimal UAV-Aided Radio Coverage Strategy for Dense Urban Areas." IEEE Transactions on Vehicular Technology 68, no. 9 (2019): 9098-9109.

[6] San-Miguel-Ayanz, J.; Durrant, T.; Boca, R.; Libertà, G.; Branco, A.; de Rigo, D.; Ferrari, D.; Maianti, P.; Artes, T.; Costa, H.; et al. Forest fires in Europe, Middle East and North Africa 2017; Joint Research Centre Technical Report, Publications Office of the European Union: Luxembourg, 2018. doi:10.2760/663443.

[7] Al-Kaff, Abdulla, Ángel Madridano, Sergio Campos, Fernando García, David Martín, and Arturo de la Escalera. "Emergency Support Unmanned Aerial Vehicle for Forest Fire Surveillance." Electronics 9, no. 2 (2020): 260.

[8] N. Arman, B. Vizvari, and G. Izbirak. "Post-earthquake response by smal UAV helicopters." Natural Hazards 80, no. 3 (2016): 1669-1688.

[9] V. Béla, M. Golabi, A. Nedjati, F. Gümüşbuğa, and G. Izbirak. "Top-down approach to design the relief system in a metropolitan city using UAV technology, part I: the first 48 h." Natural Hazards 99, no. 1 (2019): 571597.

[10] C. Nour, I. Jarkass, M. Sokhn, E. Mugellini, and O. Khaled. "Adabev: Automatic detection of abnormal behavior in video-surveillance." In International Conference on Image, Signal and Vision Computing, Oslo, Norway, pp. 172-178. 2012.

[11] S. Jonathan, J. Rodrigues, A. Alberti, P. Solic, and A. Aquino. "LoRaWAN-A low power WAN protocol for Internet of Things: A review and opportunities." In 2017 2nd International Multidisciplinary Conference on Computer and Energy Science (SpliTech), pp. 1-6. IEEE, 2017.

[12] J. Lemayian, S. Abdelhamid, S. Alturjman, E. Ever, and F. Al-Turjman. "5G in a convergent Internet of Things era: An overview." In 2018 IEEE International Conference on Communications Workshops (ICC Workshops), pp. 1-6. IEEE, 2018.

[13] W. Adrienne. "A cost-benefit analysis of Amazon Prime Air." (2015).

[14] C. Abdullah, Y. Zhang, and L. Hoover. "Police response time to inprogress burglary: A multilevel analysis." Police Quarterly 15, no. 3 (2012): 308-327.

[15] Istanbul Population. (2019-05-12). Retrieved 2019-12-16, from http://worldpopulationreview.com/world-cities/istanbul/

[16] Aktaş, Emel, Özay Özaydın, Burçin Bozkaya, Füsun Ülengin, and Şule Önsel. "Optimizing fire station locations for the Istanbul metropolitan municipality." Interfaces 43, no. 3 (2013): 240-255.

[17] Q. Bellezza, F. Grimaccia, S. Leva, M. Mussetta, and M. Aghaei. "Light Unmanned Aerial Vehicles (UAVs) for cooperative inspection of PV plants." IEEE Journal of Photovoltaics 4, no. 4 (2014): 1107-1113.

[18] Blanes i Vidal, Jordi, and Tom Kirchmaier. "The effect of police response time on crime clearance rates." The Review of Economic Studies 85, no. 2 (2017): 855-891.

[19] Xiang, Gang, Andrew Hardy, Mohammed Rajeh, and Lahari Venuthurupalli. "Design of the life-ring drone delivery system for rip current rescue." In 2016 IEEE Systems and Information Engineering Design Symposium (SIEDS), pp. 181-186. IEEE, 2016.

[20] Khan, Md Nafiz Hasan, and Carman Neustaedter. "Exploring Drones to Assist Firefighters During Emergencies." 2019.

[21] Crowder, W. Stan, and Brent E. Turvey. "False 9-1-1 Calls." In False Allegations, pp. 65-88. Academic Press, 2018.

[22] Cheng, Hsiu-Wen, Tsung-Lin Chen, and Chung-Hao Tien. "Motion Estimation by Hybrid Optical Flow Technology for UAV Landing in an Unvisited Area." Sensors 19, no. 6 (2019): 1380.

[23] Lavric, Alexandru, and Valentin Popa. "Internet of things and LoRa ${ }^{\mathrm{TM}}$ low-power wide-area networks: a survey." In 2017 International Symposium on Signals, Circuits and Systems (ISSCS), pp. 1-5. IEEE, 2017. 


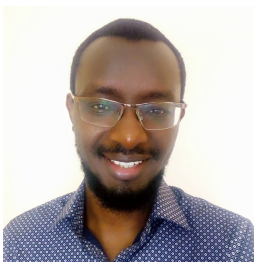

JOEL P. LEMAYIAN received the B.Sc. degree in electrical and electronics engineering from Middle East Technical University Turkey, in 2017. He is presently pursuing the master's (M.Sc.) degree in electrical and computer engineering. He is currently with Antalya Bilim University, Turkey.

He has worked as a research assistant in both Middle East Technical University and Antalya Bilim University in IoT lab and Neuroscience lab respectively. $\mathrm{He}$ is an author of numerus journals, conference papers and book chapters. His research interests include UAVs, 5G Communication networks, Artificial Intelligence, Machine Learning, and the Internet of Things (IoT) applications.

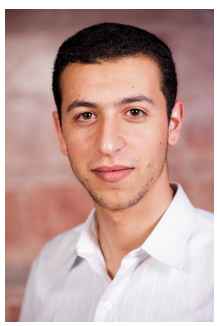

JEHAD M. HAMAMREH received the B.Sc. degree in electrical and telecommunication engineering from An-Najah University, Nablus, in 2013, and the Ph.D. degree in electricalelectronics engineering and cyber systems from Istanbul Medipol University, Turkey, in 2018. He was a Researcher with the Department of Electrical and Computer Engineering, Texas A and $\mathrm{M}$ University at Qatar. He is currently an Assistant Professor with the Electrical and Electronics Engineering Department, Antalya International (Bilim) University, Turkey.

His current research interests include wireless physical and MAC layers security, orthogonal frequency-division multiplexing multipleinput multiple-output systems, advanced waveforms design, multidimensional modulation techniques, IoT, 5G \& 6G and orthogonal/nonorthogonal multiple access schemes for future wireless systems. He is a Regular Reviewer for various refereed journals as well as a TPC Member for several international conferences. 